\title{
Effect of Helicobacter pylori Eradication on Functional Dyspepsia
}

\author{
Sung Eun Kim, ${ }^{1}$ Young Soo Park, ${ }^{1,2}$ Nayoung Kim, ${ }^{1,2 *}$ Min Soo Kim, ${ }^{1}$ Hyun Jin Jo, ${ }^{1}$ Cheol Min Shin, ${ }^{1}$ Sang Hyub Lee, \\ Jin-Hyeok Hwang, ${ }^{1,2}$ Jin-Wook Kim, ${ }^{1,2}$ Sook-Hyang Jeong, ${ }^{1,2}$ Dong Ho Lee ${ }^{1,2}$ and Hyun Chae Jung ${ }^{2}$ \\ ${ }^{1}$ Department of Internal Medicine, Seoul National University Bundang Hospital, Seongnam, Gyeonggi-do, Korea; and ${ }^{2}$ Department of Internal \\ Medicine and Liver Research Institute, Seoul National University College of Medicine, Seoul, Korea
}

\section{Background/Aims}

This study evaluated the effect of Helicobacter pylori eradication on functional dyspepsia (FD), and the relationship between the changes of histological gastritis and FD symptom responses.

\section{Methods}

A total of 213 FD patients diagnosed by Rome III criteria were consecutively enrolled. H. pylori tests and gastritis grade by the Sydney system were performed before and 1 year after the proton pump based-eradication therapy for 7 days. Serum levels of pepsinogen, and genetic polymorphisms $I L-6, I L-8$ and $I L-10$ were investigated.

\section{Results}

Total of 91 patients completed the 1 year follow-up. When the response rate of dyspepsia was compared at 1 year between the non-eradicated group $(n=24)$ and eradicated group $(n=67)$, each group showed complete response of $62.5 \%$ and $62.7 \%$; satisfactory response $(\geq 50 \%)$ of $0.0 \%$ and $19.4 \%$; partial response $(<50 \%)$ of $12.5 \%$ and $11.9 \%$; and refractory response of $25.0 \%$ and $6.0 \%$, respectively $(P=0.015)$. In addition, the responder group (complete + satisfactory response) at 1 year showed improvement of activity and chronic inflammation in both the antrum and corpus (all $P<0.001$ ). Multivariate analysis showed that $\mathrm{H}$. pylori eradication $(\mathrm{OR}, 5.81 ; 95 \% \mathrm{Cl}, 1.07-31.59)$ and symptom improvement at 3 month $(O R, 28.90 ; 95 \% \mathrm{Cl}, 5.29-157.82)$ were associated with the improvement of dyspepsia at 1 year. Among the successfully eradicated FD patients $(n=67)$, male $(P=0.013)$ and higher initial BMI $(P=0.016)$ were associated with the improvement of dyspepsia at 1 year.

\section{Conclusions}

H. pylori eradication improved FD symptoms, as well as gastritis at 1 year, suggesting that inflammation mediates FD.

(J Neurogastroenterol Motil 2013;19:233-243)

Key Words

Eradication; Functional dyspepsia; Helicobacter pylori

Received: November 21, 2012 Revised: December 14, 2012 Accepted: December 14, 2012

(c) This is an Open Access article distributed under the terms of the Creative Commons Attribution Non-Commercial License (http://creativecommons. org/licenses/by-nc/3.0) which permits unrestricted non-commercial use, distribution, and reproduction in any medium, provided the original work is properly cited.

*Correspondence: Nayoung Kim, MD

Department of Internal Medicine, Seoul National University Bundang Hospital, 82, Gumi-ro 173beon-gil, Bundang-gu, Seongnam, Gyeonggi-do 463-707, Korea

Tel: +82-31-787-7008, Fax: +82-31-787-4051, E-mail: nayoungkim49@empas.com

Sung Eun Kim and Young Soo Park equally contributed to this work.

Financial support: This research was supported from the Seoul National University Bundang Hospital research fund (Grant No. 06-2012-145).

Conflicts of interest: None.

Author contributions: Sung Eun Kim analyzed and interpreted the data and wrote the present study, and Nayoung Kim designed, organized and mediated the present study and supervised the manuscript. Min Soo Kim and Hyun Jin Jo made a questionnaire which was used in the present study. Cheol Min Shin, Sang Hyub Lee, Young Soo Park and Jin-Hyeok Hwang input and organized the data for statistical analysis. Jin-Wook Kim, Sook-Hyang Jeong and Dong Ho Lee helped analysis of data. Hyun Chae Jung supervised overall study. 


\section{Introduction}

Functional dyspepsia (FD) has been defined as a condition in which the upper abdominal symptoms occur in the absence of any organic or metabolic disease that explains the symptoms. ${ }^{1}$ Since 1998 when the first definition of FD was developed, ${ }^{2}$ numerous studies were performed to find the pathophysiological mechanisms of FD, which however have not been well established so far.

Helicobacter pylori infection is known as the main cause of chronic gastritis. ${ }^{3}$ The prevalence of $H$. pylori infection in FD patients is reported to be around $40-70 \%{ }^{4}$, therefore H. pylori-induced chronic gastric inflammation has been proposed as one of the causal factors of FD. ${ }^{5}$ Few studies have been conducted regarding the efficacy of $H$. pylori eradication on FD, which showed controversial results. ${ }^{4,6,7}$ In addition, the relationship between symptom response and gastric inflammation in FD patients, after $H$. pylori eradication, has been investigated only in a small number of studies. ${ }^{3,5}$ Even in these studies, comprehensive clinicopathological factors were not evaluated. Furthermore, it is not known which factors determine the improvement of FD symptoms in which subgroup of patients when $H$. pylori is eradicated.

From this background, the aims of this study are to evaluate the effect of $H$. pylori eradication on dyspepsia in 3 months and 1 year after $H$. pylori eradication, and to inspect the relationship between the changes in histological gastritis and responses of symptoms in FD. In addition, this study evaluated the factors associated with the improvement of dyspepsia by $H$. pylori eradication together with pepsinogen levels, genetic polymorphisms for the cytokines, environmental and host factors.

\section{Materials and Methods}

\section{Patients}

Patients, whose diagnosis was FD according to the Rome III criteria, were consecutively enrolled between 2007 and 2011, at the Seoul National University Bundang Hospital (SNUBH). They underwent gastroscopy to rule out organic diseases, such as erosive esophagitis, peptic ulcer, dysplasia, mucosa-associated lymphoid tissue lymphoma, esophageal cancer or gastric cancer. In addition, patients with history of gastric surgery, previous $H$. pylori eradication therapy, and past $H$. pylori infection ${ }^{8}$ also were excluded. This study protocol was approved by the institutional review board of SNUBH, and written informed consent was obtained from all of the participants.

\section{Questionnaire and Symptoms Assessment}

All participants completed a questionnaire under the supervision of a well-trained interviewer. The questionnaire consisted of demographic information, alcohol and smoking habit, consumption of salty and spicy food, and the Rome III criteria of FD. ${ }^{1}$ Salt and spices consumptions were graded as either low or high, according to the habit of adding salt or spices after tasting the food. The questionnaire included questions regarding dyspeptic symptoms. The participants who had positive symptoms in any one of the dyspeptic symptoms were asked to select the most bothersome symptom. Depending on the most bothersome symptom, FD was categorized into 2 types: epigastric pain syndrome or postprandial distress syndrome. The symptoms of epigastric pain syndrome were composed of epigastric pain or discomfort, and those of postprandial distress syndrome consisted of early satiety, bloating, postprandial fullness and nausea/vomiting. Body mass index (BMI) was calculated by height and weight, which were measured at the time of gastroscopy in the endoscopy department. Symptoms about irritable bowel syndrome, and history of psychotherapy or neuropsychiatric medication were also investigated from interview of the patients, as well as by medical records.

Subjects revisited for symptoms assessment at 3 months and 1 year after enrollment, regardless of eradication therapy. Responses of dyspeptic symptoms were classified into 4 sections, as follows: complete response (more than $80 \%$ of symptom resolution), satisfactory response (symptoms remained less than $50 \%$ ), partial response (symptoms remained for more than 50\%) and refractory response (unresponsive to the eradication therapy). Then, the symptom responses were classified into 2 groups: responder with complete or satisfactory subgroup, and non-responder with partial or refractory responder subgroup.

\section{Histology and Helicobacter pylori Tests}

Diagnosis of $H$. pylori was performed by histology (by modified Giemsa staining), Campylobacter-like organism (CLO) test (Delta West, Bentley, Australia) and culture, which were tested with the mucosa of antrum and corpus, respectively. ${ }^{10}$ In addition, serologic test for $H$. pylori specific immunoglobulin G ( $\mathrm{IgG}$ ) and past history of $H$. pylori eradication therapy were used to assess $H$. pylori infection status. H. pylori specific IgG was meas- 
ured by an enzyme-linked immunosorbent assay (ELISA) (Genedia H. pylori ELISA; Green Cross Medical Science Corp, Eumsung, Korea). ${ }^{11}$ If any of these 3 biopsy-based tests showed a positive result, $H$. pylori infection was defined to be current. However, when only $H$. pylori specific serum $\operatorname{IgG}$ was positive, without bacteria in the histology, culture, or CLO test, then these subjects were regarded as to have past infection of $H$. pylori. The degree of inflammatory cell infiltration, atrophy, and intestinal metaplasia was determined by H\&E staining of 4 biopsy specimens (one each from the greater curvature and lesser curvature of the antrum and corpus). Atrophy was defined as the decrease of appropriate glands. Inflammatory cell infiltration, metaplastic, and non-metaplastic atrophy were allocated, using the updated Sydney system scores, i.e., $0=$ none, $1=$ mild, $2=$ moderate and $3=$ marked. $^{12}$ The histological evaluation was performed by an experienced pathologist, unaware of the patient's details. When the specimens were not prepared well enough to correctly examine the full-thickness gastric mucosa, or whenever the inflammation interrupted a clear distinction between non- atrophic and atrophic phenotypes, the samples were determined as indefinite for atrophy and excluded from the study. ${ }^{13}$ Patients were classified as having an 'antrum predominant' or 'corpus predominant' gastritis according to chronic inflammation, depending on which score of chronic inflammation was higher in the antrum or corpus. If the scores were equal between the 2 compartments, it was classified as having a 'pangastritis' as previously described. ${ }^{10}$

\section{Genetic Polymorphisms}

Genomic DNA was isolated directly from the antral biopsy specimen. Briefly, biopsy specimens were homogenized in proteinase $\mathrm{K}$ solution $(20 \mathrm{mmol} / \mathrm{L}$ Tris- $\mathrm{HCl}$ (pH 8.0), $10 \mathrm{mmol} / \mathrm{L}$ ethylene-diamine-tetraacetic acid, $0.5 \%$ sodium dodecyl sulfate and $10 \mathrm{mg} / \mathrm{mL}$ proteinase $\mathrm{K}$ ), using a sterile micropestle, and then incubated for 3 hours at $52^{\circ} \mathrm{C}$. DNA was extracted from homogenates by phenol/chloroform extraction and ethanol precipitation. ${ }^{10,14}$ The genetic polymorphisms of IL10-592, ${ }^{15}$ IL10$1082,{ }^{16}$ IL $8-2511^{17}$ IL $8-781^{18}$ and IL6-572 ${ }^{19}$ were assessed by polymerase chain reaction restriction fragment length polymorphism (PCR-RFLP) analysis, using a Perkin Elmer model 9600 (Perkin Elmer Co., Norwalk, CT, USA). Every restriction enzyme used was purchased from New England Biolabs Inc. (Beverly, MA, USA). ${ }^{10,14}$

\section{Serology Test for Serum Pepsinogen Levels}

The measurement of serum pepsinogen I and II was con- ducted, using a latex enhanced turbidimetic immuno assay (HBi Corp, Seoul, Korea, imported from Shima Laboratories, Tokyo, Japan), and the pepsinogen I to II ratios were calculated. ${ }^{10}$ After sampling just before gastroscopy, the samples were centrifuged, immediately at $4{ }^{\circ} \mathrm{C}$, and were stored at $-70^{\circ} \mathrm{C}$ until use.

\section{Helicobacter pylori Eradication Therapy and Follow-up}

All patients with current $H$. pylori infection were recommended for eradication therapy. The patients who gave their consent for this treatment received the eradication therapy, consisting of standard dose proton pump inhibitor bid, clarithromycin $0.5 \mathrm{~g}$ bid, and amoxicillin $1 \mathrm{~g}$ bid for 7 days. H. pylori eradication was evaluated by ${ }^{13} \mathrm{C}$-urea breath test (UBiT-IR300; Otsuka Electronics, Osaka, Japan), at least 4 weeks after the completion of treatment. The breath test, using $100 \mathrm{mg}$ of ${ }^{13} \mathrm{C}$-urea was performed according to the previous report. ${ }^{20}$ The procedure with a $2.5 \%$ cut-off value has been reported to provide $97.7 \%$ sensitivity, $98.0 \%$ specificity, and $97.8 \%$ accuracy. Follow-up gastroscopy was performed 1 year after $H$. pylori eradication. Three $H$. pylori tests (modified Giemsa staining, the CLO test and culture), and histological gastritis grade by the Sydney system were also investigated, same as the baseline methods with the pathologist blinded to the initial results.

\section{Statistical Methods}

All statistical analyses were performed, using the SPSS (version 18.0; SPSS, Chicago, IL, USA). The changes in histological grade of gastritis were compared by the parametric paired $t$-test and non-parametric Wilcoxon singed-rank test. Other continuous variables were analyzed, using a Student's $t$ test. Categorical variables were analyzed using a chi-square $\left(\chi^{2}\right)$ test or Fisher's exact test. Univariate logistic regression and multivariate logistic regression were used for the analysis of risk factors, which were expressed as the odds ratio $(\mathrm{OR})$ and $95 \%$ confidence intervals $(\mathrm{CI})$. $P$-values of less than 0.05 were considered statistically significant.

\section{Results}

\section{Participant Characteristics}

Initially, 213 patients with FD were enrolled in the current study. Among FD patients, 120 patients (56.3\%) were infected with $H$. pylori, and 102 patients received $H$. pylori eradication therapy. Finally remaining 91 patients who had been followed up 
for more than 1 year were analyzed (Figure).

At first, patients with $H$. pylori (120 patients) were compared to those without $H$. pylori (93 patients). The mean age of the $H$. pylori-negative and $H$. pylori-positive patients were 48.7 and 52.1 years, respectively. There was greater proportion of women, both in the $H$. pylori-negative (65.6\%) and $H$. pylori-positive $(72.5 \%)$ groups. BMI was higher in the $H$. pylori-positive group, and the difference was statistically significant $(P=0.005)$. Pattern of gastritis was different between the $H$. pylori-positive and -negative groups $(P=0.035)$. The grades of activity and chronic inflammation of gastritis significantly increased in the $H$. pylori-positive group $(P<0.001)$, however the serum pepsinogen I/II ratio was significantly decreased in the $H$. pylori-positive group $(P<0.001)$ (Table 1$)$.

\section{Responses to Eradication Therapy}

Among $120 \mathrm{H}$. pylori-positive patients, 18 patients refused H. pylori eradication regimen. Of 102 who received $H$. pylori eradication regimen, 84 patients completed ${ }^{13} \mathrm{C}$-urea breath test, 4 weeks after the eradication therapy. Eventually, $88.1 \%$ (74 of 84 ) were found to be eradicated. When the response rate of dys- pepsia was compared at 1 year between the non-eradicated $(\mathrm{n}=$ $24)$ and eradicated $(n=67)$ group, each group showed complete response of $62.5 \%$ and $62.7 \%$; satisfactory response $(\geq 50 \%)$ of $0 \%$ and $19.4 \%$; partial response $(<50 \%)$ of $12.5 \%$ and $11.9 \%$; refractory response of $25.0 \%$ and $6.0 \%$, respectively $(P=0.015)$. The histological grades of activity $(P<0.001)$ and chronic inflammation $(P=0.010)$ in the corpus significantly decreased after H. pylori eradication (Table 2).

\section{Relationship Between Changes of Histological Gastritis and Functional Dyspepsia Symptom Improvement}

According to the responders and non-responders at 1 year, the grades of histological gastritis were evaluated in paired samples, before and after 1 year of eradication therapy (Table 3). In the responders at 1 year group, the grades of activity and chronic inflammation in the antrum and corpus significantly decreased $(P$ $<0.001)$. In the non-responders at 1 year group, activity and chronic inflammation grades $(P=0.002$ and $P=0.041$, respectively) of the corpus decreased 1 year after $H$. pylori eradication in the parametric paired $t$ test. Furthermore, there was no

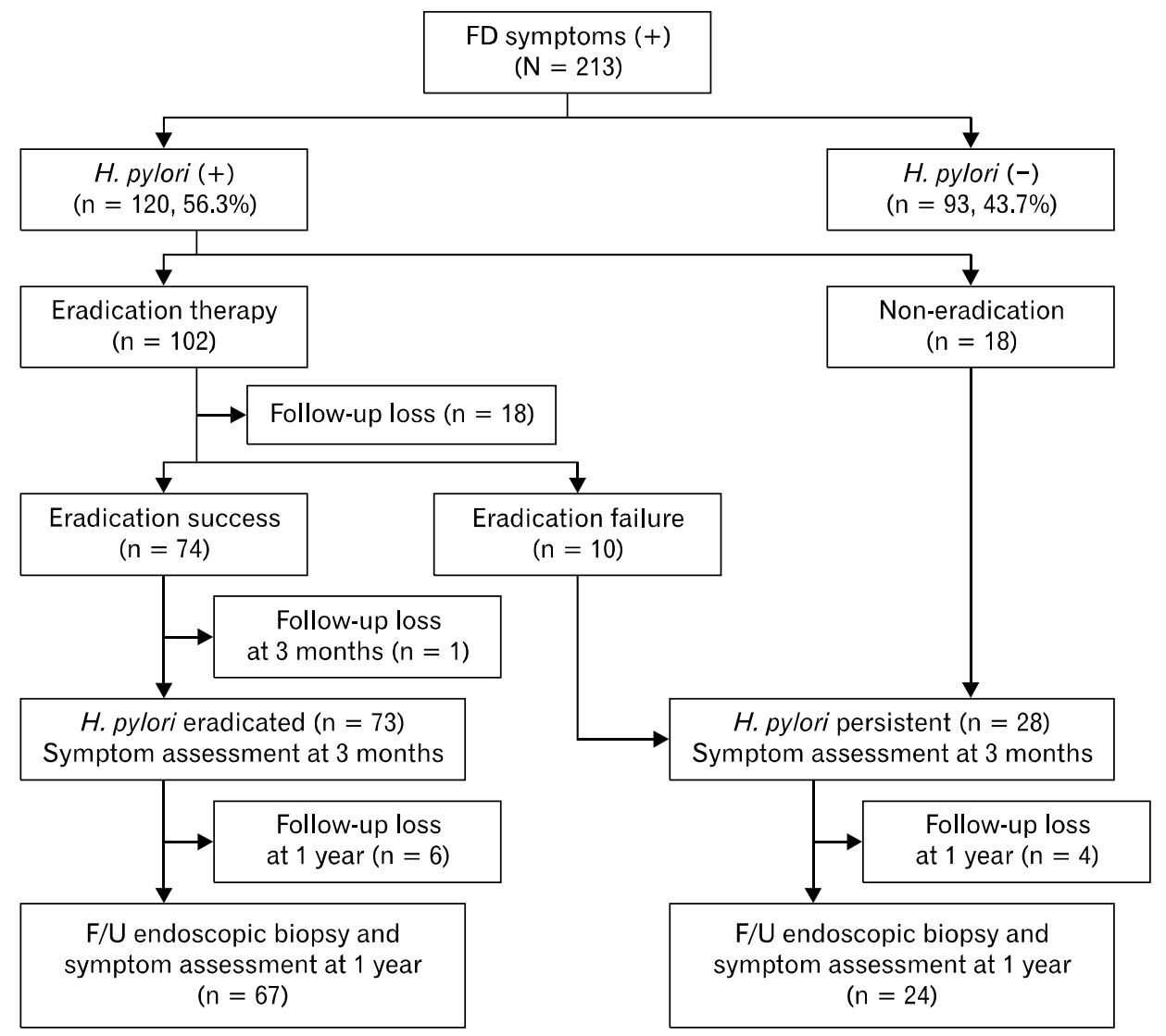

Figure. Flow of the study patients. FD, functional dyspepsia; H. pylori, Helicobacter pylori. 
Table 1. Baseline Characteristics of Helicobacter pylori-negative and Helicobacter pylori-positive Groups in Functional Dyspepsia Patients

\begin{tabular}{|c|c|c|c|}
\hline & H. pylori $(-)\left(\mathrm{n}=93^{\mathrm{a}}\right)$ & H. pylori $(+)\left(\mathrm{n}=120^{\mathrm{a}}\right)$ & $P$-value \\
\hline Age (mean $\pm \mathrm{SD}, \mathrm{yr})$ & $48.7 \pm 14.1$ & $52.1 \pm 12.8$ & 0.069 \\
\hline Gender (n [\%]) & & & 0.297 \\
\hline Male & $32 / 93(34.4)$ & $33 / 120(27.5)$ & \\
\hline Female & $61 / 93(65.6)$ & $87 / 120(72.5)$ & \\
\hline $\mathrm{BMI}\left(\right.$ mean $\left.\pm \mathrm{SD}, \mathrm{kg} / \mathrm{m}^{2}\right)$ & $22.4 \pm 2.9$ & $23.6 \pm 3.1$ & 0.005 \\
\hline Cigarette smoking (n [\%]) & $13 / 92(14.1)$ & $10 / 120(8.3)$ & 0.189 \\
\hline Alcohol intake (n [\%]) & $54 / 92(58.7)$ & $69 / 120(57.5)$ & 0.889 \\
\hline Salty food (n $[\%])$ & & & 0.628 \\
\hline Low & $24 / 88(27.3)$ & 28/117 (23.9) & \\
\hline High & $64 / 88(72.7)$ & $89 / 117(76.1)$ & \\
\hline Spicy food (n [\%]) & & & 0.269 \\
\hline Low & $20 / 88(22.7)$ & $35 / 117(29.9)$ & \\
\hline High & 68/88 (77.3) & $82 / 117(70.1)$ & \\
\hline $\operatorname{IBS}(\mathrm{n}[\%])$ & $17 / 93(18.3)$ & $18 / 120(15.0)$ & 0.578 \\
\hline NP follow-up or NP medication (n [\%]) & $11 / 93(11.8)$ & $16 / 120(13.3)$ & 0.837 \\
\hline The most bothersome symptoms (n [\%]) & & & 0.728 \\
\hline Heartburn/chest pain & $21 / 93(22.6)$ & $27 / 120(22.5)$ & \\
\hline Acid regurgitation & $13 / 93(14.0)$ & $11 / 120(9.2)$ & \\
\hline Globus sensation & $15 / 93(16.1)$ & $23 / 120(19.2)$ & \\
\hline Upper abdominal pain & $14 / 93(15.1)$ & $21 / 120(17.5)$ & \\
\hline Early satiety & $2 / 93(2.2)$ & $0 / 120(0.0)$ & \\
\hline Bloating & $13 / 93(14.0)$ & $17 / 120(14.2)$ & \\
\hline Postprandial fullness & $8 / 93(8.6)$ & $13 / 120(10.8)$ & \\
\hline Nausea/vomiting & $7 / 93(7.5)$ & $8 / 120(6.7)$ & \\
\hline EPS:PDS (n [\%]) & $63: 30(67.7: 32.3)$ & $82: 38(68.3: 31.7)$ & 1.000 \\
\hline Patterns of gastritis (n [\%]) & & & 0.035 \\
\hline Antrum-predominant gastritis & $3 / 80(3.8)$ & $13 / 110(11.8)$ & \\
\hline Corpus-predominant gastritis & $8 / 80(10.0)$ & $19 / 110(17.3)$ & \\
\hline Pangastritis & $69 / 80(86.3)$ & $78 / 110(70.9)$ & \\
\hline \multicolumn{4}{|l|}{ Antrum $($ mean $\pm \mathrm{SD})$} \\
\hline Activity & $0.12 \pm 0.37$ & $1.72 \pm 0.71$ & $<0.001$ \\
\hline Chronic inflammation & $1.11 \pm 0.32$ & $2.06 \pm 0.47$ & $<0.001$ \\
\hline Atrophy & $0.23 \pm 0.52$ & $0.65 \pm 0.65$ & $<0.001$ \\
\hline Intestinal metaplasia & $0.22 \pm 0.52$ & $0.49 \pm 0.78$ & 0.005 \\
\hline \multicolumn{4}{|l|}{ Corpus $($ mean $\pm \mathrm{SD})$} \\
\hline Activity & $0.15 \pm 0.45$ & $1.88 \pm 0.71$ & $<0.001$ \\
\hline Chronic inflammation & $1.18 \pm 0.41$ & $2.13 \pm 0.45$ & $<0.001$ \\
\hline Atrophy & $0.18 \pm 0.52$ & $0.44 \pm 0.69$ & 0.008 \\
\hline Intestinal metaplasia & $0.16 \pm 0.46$ & $0.27 \pm 0.60$ & 0.164 \\
\hline \multicolumn{4}{|l|}{ Genetic polymorphism } \\
\hline IL10-592(A/A:C/A:C/C $)^{\mathrm{b}}$ & $32: 28: 6$ & $43: 37: 14$ & 0.550 \\
\hline IL10-1082 (A/A:G/A:G/G) & $59: 6: 1$ & $79: 14: 0$ & 0.274 \\
\hline IL8-251 (T/T:A/T:A/A $)^{\mathrm{b}}$ & $31: 25: 11$ & $43: 36: 16$ & 0.992 \\
\hline IL8-781 (C/C:C/T:T/T) $)^{\mathrm{b}}$ & $35: 23: 7$ & $56: 29: 10$ & 0.795 \\
\hline IL6-572 (C/C:G/C:G/G) & $34: 28: 4$ & $46: 40: 6$ & 0.980 \\
\hline Pepsinogen I/II ratio (mean $\pm \mathrm{SD}$ ) & $3.9 \pm 2.2$ & $2.8 \pm 1.9$ & $<0.001$ \\
\hline
\end{tabular}

${ }^{a}$ Total number of each group (missing values were not included and the number behind the dash is the total number of subjects who answered each question), ${ }^{\mathrm{b}}$ Insufficient samples were not included in genetic polymorphism.

BMI, body mass index; IBS, irritable bowel syndrome; NP, neuropsychiatry; EPS, epigastric pain syndrome; PDS, postprandial distress syndrome; H. pylori, Helicobacter pylori. 
Table 2. Main Study Outcomes of Helicobacter pylori-persistent and Helicobacter pylori-eradicated Groups in Functional Dyspepsia Patients

\begin{tabular}{|c|c|c|c|}
\hline & H. pylori-persistent $\left(\mathrm{n}=28^{\mathrm{a}}\right)$ & H. pylori-eradicated $\left(\mathrm{n}=74^{\mathrm{a}}\right)$ & $P$-value \\
\hline Responses at 3 months (n [\%]) & & & 0.124 \\
\hline Complete & $18 / 28(64.3)$ & $37 / 73(50.7)$ & \\
\hline Satisfactory & $2 / 28(7.1)$ & $16 / 73(21.9)$ & \\
\hline Partial & $3 / 28(10.7)$ & $14 / 73(19.2)$ & \\
\hline Refractory & $5 / 28(17.9)$ & $6 / 73(8.2)$ & \\
\hline Responses at 1 year (n [\%]) & & & 0.015 \\
\hline Complete & $15 / 24(62.5)$ & $42 / 67(62.7)$ & \\
\hline Satisfactory & $0 / 24(0)$ & $13 / 67(19.4)$ & \\
\hline Partial & $3 / 24(12.5)$ & $8 / 67(11.9)$ & \\
\hline Refractory & $6 / 24(25.0)$ & $4 / 67(6.0)$ & \\
\hline Responses at 3 months (n [\%]) & & & 1.000 \\
\hline Responder & $20 / 28(71.4)$ & $53 / 73(72.6)$ & \\
\hline Non-responder & $8 / 28(28.6)$ & $20 / 73(27.4)$ & \\
\hline Responses at 1 year (n [\%]) & & & 0.087 \\
\hline Responder & $15 / 24(62.5)$ & $55 / 67(82.1)$ & \\
\hline Non-responder & $9 / 24(37.5)$ & $12 / 67(17.9)$ & \\
\hline Patterns of gastritis after eradication therapy (n [\%]) & & & 0.256 \\
\hline Antrum-predominant gastritis & $0 / 4(0.0)$ & $5 / 30(16.7)$ & \\
\hline Corpus-predominant gastritis & $2 / 4(50.0)$ & $6 / 30(16.7)$ & \\
\hline Pangastritis & $2 / 4(50.0)$ & $19 / 30(66.7)$ & \\
\hline Antrum after eradication therapy (mean $\pm \mathrm{SD})$ & $(n=4)$ & $(\mathrm{n}=30)$ & \\
\hline Activity & $0.75 \pm 0.96$ & $0.43 \pm 0.63$ & 0.377 \\
\hline Chronic inflammation & $1.75 \pm 0.50$ & $1.40 \pm 0.50$ & 0.196 \\
\hline Atrophy ${ }^{\mathrm{b}}$ & $0.50 \pm 0.71$ & $0.47 \pm 0.61$ & 0.955 \\
\hline Intestinal metaplasia & $0.50 \pm 0.58$ & $0.43 \pm 0.57$ & 0.827 \\
\hline Corpus after eradication therapy (mean $\pm \mathrm{SD})$ & $(\mathrm{n}=4)$ & $(\mathrm{n}=30)$ & \\
\hline Activity & $1.75 \pm 0.50$ & $0.43 \pm 0.63$ & $<0.001$ \\
\hline Chronic inflammation & $2.25 \pm 0.50$ & $1.43 \pm 0.57$ & 0.010 \\
\hline Atrophy ${ }^{\mathrm{b}}$ & $1.00 \pm 1.00$ & $0.22 \pm 0.60$ & 0.059 \\
\hline Intestinal metaplasia & $0.75 \pm 0.96$ & $0.37 \pm 0.67$ & 0.312 \\
\hline
\end{tabular}

${ }^{a}$ Total number of each group (missing values were not included and the number behind the dash is the total number of subjects who answered each question), ${ }^{\mathrm{b}}$ Inappropriate specimens were not included.

Responder included patients who showed complete and satisfactory response.

H. pylori, Helicobacter pylori.

statistical difference in the atrophy and intestinal metaplasia grades of the antrum and corpus, before and 1 year after the eradication therapy in the 2 groups.

\section{Predictive Factors for Improvement of Functional Dyspepsia}

The predictive factors for improvement of dyspeptic symptoms at 1 year are revealed in Table 4 . In a univariate analysis, male gender $(P=0.028)$, higher BMI before enrollment ( $P=$ $0.002)$, the history of psychotherapy or neuropsychiatric medication $(P=0.009)$, and responders at 3 months $(P<0.001)$ were related with the improvement of dyspeptic symptoms at 1 year. Multivariate analysis showed that responders at 3 months (OR, 28.90; 95\% CI, 5.29-157.82) and H. pylori eradicated status (OR, 5.81; 95\% CI, 1.07-31.59) were associated with the improvement of dyspeptic symptoms at 1 year.

\section{Predictive Factors for Improvement of Functional Dyspepsia After Successful Helicobacter pylori Eradication}

Among the successful $H$. pylori eradicated group $(\mathrm{n}=67)$, 12 patients were classified as non-responders (17.9\%), and 55 were classified as responders $(82.1 \%)$. The male gender $(P=$ $0.013)$ and higher initial BMI $(P=0.010)$ were associated with 
Table 3. Relation Between Changes of Histological Gastritis and Symptom Improvement of Functional Dyspepsia

\begin{tabular}{|c|c|c|c|c|c|c|c|c|c|c|}
\hline & \multicolumn{5}{|c|}{ Non-responders at 1 year $\left(\mathrm{n}=8^{\mathrm{a}, \mathrm{b}}\right)$} & \multicolumn{5}{|c|}{ Responders at 1 year $\left(\mathrm{n}=26^{\mathrm{a}, \mathrm{b}}\right)$} \\
\hline & $\begin{array}{c}\text { Before } \\
\text { eradication } \\
\text { therapy } \\
\text { (mean } \pm \mathrm{SD})\end{array}$ & $\begin{array}{c}\text { After } \\
\text { eradication } \\
\text { therapy } \\
\text { (mean } \pm \mathrm{SD})\end{array}$ & $P$-value ${ }^{\mathrm{d}}$ & $Z^{\mathrm{e}, \mathrm{f}}$ & $P$-value & $\begin{array}{c}\text { Before } \\
\text { eradication } \\
\text { therapy } \\
(\text { mean } \pm \mathrm{SD})\end{array}$ & $\begin{array}{c}\text { After } \\
\text { eradication } \\
\text { therapy } \\
(\text { mean } \pm \mathrm{SD})\end{array}$ & $P$-value ${ }^{\mathrm{d}}$ & $Z^{\mathrm{e}, \mathrm{f}}$ & $P$-value ${ }^{\mathrm{e}}$ \\
\hline \multicolumn{11}{|l|}{ Antrum } \\
\hline Activity & $1.00 \pm 1.07$ & $0.25 \pm 0.46$ & 0.170 & -1.622 & 0.105 & $1.62 \pm 0.85$ & $0.46 \pm 0.65$ & $<0.001$ & -3.798 & $<0.001$ \\
\hline Chronic inflammation & $1.75 \pm 0.71$ & $1.38 \pm 0.52$ & 0.285 & -1.134 & 0.257 & $2.04 \pm 0.53$ & $1.50 \pm 0.51$ & $<0.001$ & -3.300 & 0.001 \\
\hline Atrophy ${ }^{c}$ & $0.75 \pm 0.96$ & $0.50 \pm 0.58$ & 0.718 & -0.447 & 0.655 & $0.58 \pm 0.67$ & $0.25 \pm 0.45$ & 0.220 & -1.265 & 0.206 \\
\hline Intestinal metaplasia & $0.75 \pm 0.89$ & $0.63 \pm 0.52$ & 0.685 & -0.447 & 0.655 & $0.58 \pm 0.81$ & $0.35 \pm 0.56$ & 0.136 & -1.513 & 0.130 \\
\hline \multicolumn{11}{|l|}{ Corpus } \\
\hline Activity & $2.13 \pm 0.35$ & $0.50 \pm 0.76$ & 0.002 & -2.414 & 0.016 & $1.92 \pm 0.69$ & $0.62 \pm 0.75$ & $<0.001$ & -3.926 & $<0.001$ \\
\hline Chronic inflammation & $2.25 \pm 0.46$ & $1.38 \pm 0.74$ & 0.041 & -1.933 & 0.053 & $2.35 \pm 0.49$ & $1.58 \pm 0.58$ & $<0.001$ & -4.082 & $<0.001$ \\
\hline Atrophy ${ }^{c}$ & $0.29 \pm 0.49$ & $0.43 \pm 0.79$ & 0.736 & -0.378 & 0.705 & $0.63 \pm 0.81$ & $0.31 \pm 0.70$ & 0.237 & -1.155 & 0.248 \\
\hline Intestinal metaplasia & $0.00 \pm 0.00$ & $0.25 \pm 0.46$ & 0.170 & -1.414 & 0.157 & $0.31 \pm 0.62$ & $0.46 \pm 0.76$ & 0.356 & -0.921 & 0.357 \\
\hline
\end{tabular}

${ }^{a}$ Total number of patients, who were performed gastritis grade both before and after eradication therapy by the Sydney system; ${ }^{b} 12(57.1 \%)$ and 55 (78.6\%) were eradicated in non-responders and responders at 1 year, respectively; ${ }^{c}$ Inappropriate specimens were not included; ${ }^{\mathrm{d} V a r i a b l e s ~ w e r e ~ c o m p a r e d ~ u s i n g ~ t h e ~ p a r a m e t r i c ~ p a i r e d ~}$ $t$ test and a $P$-value $<0.05$ was considered significant; ${ }^{e}$ Variables were compared using the non-parametric Wilcoxon signed-rank test and a $P$-value $<0.05$ was considered significant; ${ }^{\mathrm{f}} \mathrm{Z}$ means after eradication therapy minus before eradication therapy.

Table 4. Factors Predicting Symptoms Improvement in Functional Dyspepsia

\begin{tabular}{|c|c|c|c|c|c|}
\hline & $\begin{array}{l}\text { Non-responders } \\
\text { at } 1 \text { year }(\mathrm{n}=21)\end{array}$ & $\begin{array}{l}\text { Responders } \\
\text { at } 1 \text { year }(\mathrm{n}=70)\end{array}$ & $\begin{array}{c}\text { Univariated } \\
P \text {-value }\end{array}$ & $\begin{array}{c}\text { Multivariated } \\
P \text {-value }\end{array}$ & $\begin{array}{c}\text { Adjusted } \\
\text { OR }(95 \% \mathrm{CI})^{\mathrm{a}}\end{array}$ \\
\hline Age $($ mean $\pm \mathrm{SD}, \mathrm{yr})$ & $52.4 \pm 15.0$ & $52.9 \pm 11.8$ & 0.870 & 0.891 & $1.00(0.94-1.06)$ \\
\hline \multicolumn{6}{|l|}{ Gender (n [\%]) } \\
\hline Female & $19(29.7)$ & $45(70.3)$ & & & \\
\hline Male & $2(7.4)$ & $25(92.6)$ & 0.028 & 0.062 & $10.65(0.89-127.46)$ \\
\hline $\mathrm{BMI}\left(\right.$ mean $\left.\pm \mathrm{SD}, \mathrm{kg} / \mathrm{m}^{2}\right)$ & $21.7 \pm 2.7$ & $24.2 \pm 3.2$ & 0.002 & 0.225 & $1.20(0.89-1.61)$ \\
\hline \multicolumn{6}{|l|}{ IBS (n [\%]) } \\
\hline No & $18(22.8)$ & $61(77.2)$ & & & \\
\hline Yes & $3(25.0)$ & $9(75.0)$ & 1.000 & 0.056 & $14.94(0.94-238.23)$ \\
\hline \multicolumn{6}{|c|}{ NP follow-up or NP medication (n [\%]) } \\
\hline No & $13(17.3)$ & $62(82.7)$ & & & \\
\hline Yes & $8(50.0)$ & $8(50.0)$ & 0.009 & 0.145 & $0.25(0.04-1.62)$ \\
\hline \multicolumn{6}{|c|}{ Responses at 3 months (n [\%]) } \\
\hline Non-responder & $15(60.0)$ & $10(40.0)$ & & & \\
\hline Responder & $6(9.1)$ & $60(90.9)$ & $<0.001$ & $<0.001$ & $28.90(5.29-157.82)$ \\
\hline \multicolumn{6}{|l|}{ H. pylori eradication (n [\%]) } \\
\hline H. pylori persistent & $9(37.5)$ & $15(62.5)$ & & & \\
\hline H. pylori eradicated & $12(17.9)$ & $55(82.1)$ & 0.087 & 0.042 & $5.81(1.07-31.59)$ \\
\hline
\end{tabular}

${ }^{a}$ Logistic model including terms of age, gender, BMI, the presence of IBS, the history of received psychotherapy or treated neuropsychiatric medication, the responses at 3 months, and $H$. pylori status after eradication.

BMI, body mass index; IBS, irritable bowel syndrome; NP, neuropsychiatry; H. pylori, Helicobacter pylori.

symptoms response at 1 year in a univariate analysis. As there was no male gender in the non-responder group, calculation of OR was impossible in a multivariate analysis. Finally, only higher ini- tial BMI (OR, 1.47; 95\% CI, 1.07-2.01) was associated with symptoms response at 1 year in a multivariate analysis (Table 5 ). 
Table 5. Factors Predicting Symptoms Improvement in Functional Dyspepsia after Successful Helicobacter pylori Eradication

\begin{tabular}{|c|c|c|c|c|c|}
\hline & $\begin{array}{l}\text { Non-responders at } 1 \text { year } \\
\qquad\left(\mathrm{n}=12^{\mathrm{a}}\right)\end{array}$ & $\begin{array}{l}\text { Responders at } 1 \text { year } \\
\qquad\left(\mathrm{n}=55^{\mathrm{a}}\right)\end{array}$ & $\begin{array}{l}\text { Univariated } \\
P \text {-value }\end{array}$ & $\begin{array}{c}\text { Multivariated } \\
P \text {-value }\end{array}$ & $\begin{array}{c}\text { Adjusted } \\
\text { OR }(95 \% \mathrm{CI})^{\mathrm{d}}\end{array}$ \\
\hline Age (mean $\pm \mathrm{SD}, \mathrm{yr})$ & $50.2 \pm 12.6$ & $52.6 \pm 11.0$ & 0.511 & 0.719 & $0.99(0.93-1.05)$ \\
\hline \multicolumn{6}{|l|}{ Gender (n [\%]) } \\
\hline Female & $12(25.5)$ & $35(74.5)$ & & & \\
\hline Male & $0(0.0)$ & $20(100.0)$ & $0.013^{\mathrm{c}}$ & - & - \\
\hline $\mathrm{BMI}\left(\right.$ mean $\left.\pm \mathrm{SD}, \mathrm{kg} / \mathrm{m}^{2}\right)$ & $22.0 \pm 1.9$ & $24.3 \pm 2.8$ & 0.010 & 0.018 & $1.46(1.07-2.00)$ \\
\hline \multicolumn{6}{|l|}{ IBS (n [\%]) } \\
\hline No & $11(19.0)$ & $47(81.0)$ & & & \\
\hline Yes & $1(11.1)$ & $8(88.9)$ & 1.000 & - & - \\
\hline \multicolumn{6}{|c|}{ NP follow-up or NP medication (n [\%]) } \\
\hline No & $8(14.0)$ & $49(86.0)$ & & & \\
\hline Yes & $4(40.0)$ & $6(60.0)$ & 0.070 & 0.177 & $0.30(0.05-1.73)$ \\
\hline Cigarette smoking (n [\%]) & $0 / 12(0.0)$ & $6 / 55(100.0)$ & 0.582 & - & - \\
\hline Alcohol intake (n [\%]) & $6 / 12(15.8)$ & $32 / 55(84.2)$ & 0.750 & - & - \\
\hline \multicolumn{6}{|l|}{ Salty food (n [\%]) } \\
\hline Low & $1 / 11(6.7)$ & $14 / 53(93.3)$ & & & \\
\hline High & $10 / 11(20.4)$ & $39 / 53(79.6)$ & 0.434 & - & - \\
\hline \multicolumn{6}{|l|}{ Spicy food (n [\%]) } \\
\hline Low & $2 / 11(10.5)$ & $17 / 52(89.5)$ & & & \\
\hline High & $9 / 11(20.5)$ & $35 / 52(79.5)$ & 0.480 & - & - \\
\hline \multicolumn{6}{|l|}{ EPS or PDS (n [\%]) } \\
\hline EPS & $10(20.8)$ & $38(79.2)$ & & & \\
\hline PDS & $2(10.5)$ & $17(89.5)$ & 0.485 & - & - \\
\hline \multicolumn{6}{|l|}{ Patterns of gastritis (n [\%]) } \\
\hline Antrum-predominant gastritis & $0 / 10(0.0)$ & $6 / 52(100.0)$ & & & \\
\hline Corpus-predominant gastritis & $2 / 10(20.0)$ & $8 / 52(80.0)$ & & & \\
\hline Pangastritis & 8/10 (17.4) & $38 / 52(82.6)$ & 0.517 & - & - \\
\hline \multicolumn{6}{|l|}{ Genetic polymorphism } \\
\hline IL10-592 (A/A:C/A:C/C $)^{\mathrm{b}}$ & $5: 2: 2$ & $17: 18: 7$ & 0.516 & - & - \\
\hline IL10-1082 (A/A:G/A:G/G) & $8: 1: 0$ & $34: 7: 0$ & 1.000 & - & - \\
\hline IL8-251 (T/T:A/T:A/A $)^{\mathrm{b}}$ & $4: 3: 2$ & $24: 15: 5$ & 0.666 & - & - \\
\hline IL8-781 (C/C:C/T:T/T) ${ }^{\mathrm{b}}$ & $6: 1: 2$ & $30: 11: 3$ & 0.284 & - & - \\
\hline IL6-572 (C/C:G/C:G/G) $)^{\mathrm{b}}$ & $2: 5: 1$ & $20: 19: 5$ & 0.542 & - & - \\
\hline Pepsinogen $\mathrm{I} / \mathrm{II}$ ratio $($ mean $\pm \mathrm{SD})$ & $2.2 \pm 1.0$ & $2.6 \pm 1.5$ & 0.411 & - & - \\
\hline
\end{tabular}

${ }^{\mathrm{a}}$ Total number of each group (missing values were not included and the number behind the dash is the total number of subjects who answered each question); ${ }^{\mathrm{b}}$ Insufficient samples were not included in genetic polymorphism; ${ }^{\mathrm{c}}$ Variables were compared using the non-parametric Fisher's exact test and a $P$-value $<0.05$ was considered significant; ${ }^{\mathrm{d}}$ Logistic model including terms of age, BMI and the history of received psychotherapy or treated neuropsychiatric medication.

BMI, body mass index; IBS, irritable bowel syndrome; NP, neuropsychiatry; EPS, epigastric pain syndrome; PDS, postprandial distress syndrome; H. pylori, Helicobacter pylori.

\section{Discussion}

H. pylori eradication is recommended not only for $\mathrm{FD}$, but also for all $H$. pylori infection in Japan, ${ }^{21}$ and the American Gastroenterological Association also recommended H. pylori test, and to treat it as the initial management strategy for FD patients, younger than 55 years if the prevalence of infection is $>10 \%{ }^{22}$
There have been only 2 studies regarding the effectiveness of $H$. pylori eradication therapy on FD patients in Korea so far, which showed quite different results. ${ }^{23,24}$ Recently, a randomized, double-blind, placebo-controlled study was attempted to investigate the clinical usefulness of $H$. pylori eradication therapy in Korea, ${ }^{25}$ but it failed because only a few patients were eligible for the including criteria. With this background, we planned to investigate the responses of dyspeptic symptoms, according to $H$. pylori erad- 
ication in FD patients.

Interestingly, successful $H$. pylori eradication was found to be the most important factor in the improvement of dyspeptic symptoms, at 1 year follow-up among the comprehensive variables in the current study. This result is in agreement with the other reports from Korea, ${ }^{23}$ Japan, ${ }^{26}$ as well as from Singapore. ${ }^{6}$ Successful $H$. pylori eradication improved the quality of life of FD patients, especially $H$. pylori-positive patients with ulcer-like FD or dysmotility-like FD from the study in Japan. ${ }^{26}$ The study of Singapore nicely demonstrated that a 13 -fold increased chance of FD symptom resolution was found in the successful $H$. pylori eradication FD patients compared to the H. pylori persisted (95\% CI, 1.10-17.70). ${ }^{6}$ Recent systemic review and meta-analysis from China also reported that the summary OR for improvement in FD patients after $H$. pylori eradication was 3.61 (95\% CI, 2.62-4.98). ${ }^{4}$ In contrast, no convincing evidence was found that successful eradication of $H$. pylori infection relieved the symptoms of FD patients at 12 months after the treatment in Optimal Regimen Cures Helicobacter Induced Dyspepsia study, which was mainly performed in the West. ${ }^{27}$ These regional differences could have originated from the different prevalence of the putative pathogenic factors and virulent $H$. pylori strains. ${ }^{6}$ Several articles suggested that $H$. pylori-positive FD could be considered as a kind of an organic disease and as a different disease entity from FD. ${ }^{28-30}$ According to our study, it would be tenable that $H$. pylori eradication therapy is not effective in all FD patients with $H$. pylori-positive, but is effective in a subset of $\mathrm{FD}$ patients with $H$. pylori-positive.

In terms of dyspeptic symptoms, there was no significant difference in the response rate of dyspepsia at 3 months between the non-eradicated and eradicated groups. The result of a short term (6-12 weeks) follow-up assessment is similar with that of other studies. ${ }^{6,7}$ Japanese study with 12 week follow-up did not show any significant difference in dyspeptic symptoms scores between H. pylori eradication therapy and placebo groups. ${ }^{7}$ In contrast, a longer follow-up study, such as in Singapore, ${ }^{6}$ showed a definite improvement of dyspeptic symptom. The reason of this statistical difference between the short and long-term follow-up after eradication is not well clarified. However, it could be related with the dynamic change of gastric secretion. Ijima et $\mathrm{al}^{31}$ reported that atrophy score in the antrum and body did not change significantly in patients after $H$. pylori eradication. However, the effects of $H$. pylori eradication on the gastric acid secretion in patients with peptic ulcer using endoscopic gastrin test showed that it took several months for the decrease of acid secretion after ending the tro- phic effect of gastrin. ${ }^{31}$ This stabilization of acid secretion could be a clue for the difference in dyspeptic symptom responses between 3 months and 1 year after $H$. pylori eradication.

Another issue is about the predictive factors of the symptoms response of $\mathrm{FD}$ patients after successful $H$. pylori eradication. In this study, male gender and higher initial BMI were related with the improvement of dyspepsia at 1 year. When we compared the non-responders and responders groups at 1 year in 67 patients with successful $H$. pylori eradicated group, gender difference was not able to be analyzed by logistic regression analysis mainly because there was no male patient. FD was more common in the female than male generally ${ }^{32}$; similarly, the proportion of female gender was higher than male gender in our study. It is generally recognized that psychological co-morbidity is less common in the male gender than in women. ${ }^{33}$ Thus, FD male gender could be less influenced by those factors which are often related with $\mathrm{FD}^{34}$ It is very interesting that higher initial BMI was found to be another factor. A few studies reported that BMI could be increased after $H$. pylori eradication therapy by way of ghrelin. ${ }^{35,36}$ However, previous Korean study, including our hospital, ${ }^{37}$ did not show any significant BMI change after successful $H$. pylori eradication as in this study. Interestingly, a German study reported that higher BMI was related with the placebo responders than with the placebo non-responders in FD patients $(P=0.035) .{ }^{38}$ The reason of the association between higher BMI and the symptoms response in FD patients after successful $H$. pylori eradication is difficult to interpret, and further studies will be necessary.

There have been several studies that evaluated the affecting factors in the improvement of dyspeptic symptoms. To our knowledge, there was no previous study that comprehensively examined the factors between $H$. pylori infection and dyspepsia, including genetic polymorphisms of cytokines and pepsinogen. A recent study from our hospital has shown that genetic polymorphisms of these cytokines, such as $I L-10, I L-8$ and $I L-6$, were associated with the development of $H$. pylori-associated gastroduodenal diseases. ${ }^{14}$ However, these factors did not show any statistically significant relationship with FD. G-protein $\beta 3$ subunit gene polymorphism (C825T), serotonin transporter promoter gene polymorphism, serotonin transporter SL genotype, $I L-17 F$ and migration inhibitory factor were found to be associated with the development of dyspeptic symptoms. ${ }^{39}$ In terms of specific $H$. pylori antigens, several studies evaluated the association of $H$. pylori virulence markers cytotoxin-associated gene $(\operatorname{cag} A)$ or vacuolating-associated gene (vacA) in patients with dys- 
peptic symptoms. ${ }^{40-42}$ The presence of $H$. pylori virulence factors was not predictive for organic diseases or FD. ${ }^{41,42}$ However, by a study from Pakistan, the higher degree of inflammation was reported to be related with the $H$. pylori cagAs1am1, cagAs1bm1, vacAs $1 a m 1$ and vacAs $1 b m 1$ in patients with gastroduodenal diseases including FD. ${ }^{40}$ This study concluded that vacA allele's s1am 1 and $s 1 b m 1$ were associated with $H$. pylori associated diseases and inflammation. Thus, further studies are necessary for the role of genetic polymorphisms and $H$. pylori virulence markers in FD patients.

The decrease of the serum pepsinogen I/II ratio is a marker of atrophic gastritis, ${ }^{43}$ and $H$. pylori-induced gastritis progresses to gastric atrophy. ${ }^{44}$ There was a significant correlation between gastric emptying and pepsinogen I/II ratio in the $H$. pylori-positive patients with FD. ${ }^{45}$ However, the degree of atrophy of gastric mucosa was not different between the responders and non-responders at 1 year. Instead, activity and chronic inflammation in the antrum, as well as in the corpus significantly improved in the responders, but not in the non-responders, suggesting that H. pylori-induced inflammation mediates dyspeptic symptoms, but not atrophy itself. Our results could be supported by the report that the activity and inflammation increased with increase of H. pylori intensity in patients with dyspepsia. ${ }^{46}$ In addition, $H$. $p y$ lori-positive FD patients with 'antrum predominant' chronic gastritis showed symptom improvement at 1 year after the successful eradication. ${ }^{3}$ Taken together, the improvement of dyspepsia after successful $H$. pylori eradication might be associated with the decrease of inflammation, which the effect could be bigger in male and in FD patients with higher BMI.

This study has some limitations. First, too many variables were tested for a small study population. This could weaken the statistical power of multivariate analysis. Second, patients were allowed to know the result of $H$. pylori eradication therapy, which might have influenced the symptoms in some participants. However, there was no significant difference in symptom responses at 3 months between the $H$. pylori-eradicated and persistent groups. Therefore, knowing the result of $H$. pylori eradication may not have worked on the response of dyspeptic symptoms at 1 year.

In conclusion, $H$. pylori eradication was the only important factor in the FD improvement at 1 year, which accompanied the improvement of inflammation in the antrum and corpus. Our results suggest that inflammation is one of the most important factors that mediate FD.

\section{References}

1. Tack J, Talley NJ, Camilleri M, et al. Functional gastroduodenal disorders. Gastroenterology 2006;130:1466-1479.

2. Colin-Jones D, Bloom B, Bodemar G, et al. Management of dyspepsia: report of a working party. Lancet 1988;1:576-579.

3. Koskenpato J, Färkkilä M, Sipponen P. Helicobacter pylori and different topographic types of gastritis: treatment response after successful eradication therapy in functional dyspepsia. Scand J Gastroenterol 2002;37:778-784

4. Jin X, Li YM. Systematic review and meta-analysis from Chinese literature: the association between Helicobacter pylori eradication and improvement of functional dyspepsia. Helicobacter 2007;12:541546.

5. Turkkan E, Uslan I, Acarturk G, et al. Does Helicobacter pylori-induced inflammation of gastric mucosa determine the severity of symptoms in functional dyspepsia? J Gastroenterol 2009;44:66-70.

6. Gwee KA, Teng L, Wong RK, Ho KY, Sutedja DS, Yeoh KG. The response of Asian patients with functional dyspepsia to eradication of Helicobacter pylori infection. Eur J Gastroenterol Hepatol 2009;21: 417-424.

7. Miwa H, Hirai S, Nagahara A, et al. Cure of Helicobacter pylori infection does not improve symptoms in non-ulcer dyspepsia patients-a double-blind placebo-controlled study. Aliment Pharmacol Ther 2000;14:317-324.

8. Lee JH, Kim N, Chung JI, et al. Long-term follow up of Helicobacter pylori IgG serology after eradication and reinfection rate of $H$. pylori in South Korea. Helicobacter 2008;13:288-294.

9. Lee ES, Kim N, Lee SH, et al. Comparison of risk factors and clinical responses to proton pump inhibitors in patients with erosive oesophagitis and non-erosive reflux disease. Aliment Pharmacol Ther 2009;30:154-164.

10. Kang JM, Kim N, Shin CM, et al. Predictive factors for improvement of atrophic gastritis and intestinal metaplasia after Helicobacter pylori eradication: a three-year follow-up study in Korea. Helicobacter 2012;17:86-95.

11. Kim SY, Ahn JS, Ha YJ, et al. Serodiagnosis of Helicobacter pylori infection in Korean patients using enzyme-linked immunosorbent assay. J Immunoassay 1998;19:251-270.

12. Dixon MF, Genta RM, Yardley JH, Correa P. Classification and grading of gastritis. The updated Sydney System. International Workshop on the Histopathology of Gastritis, Houston 1994. Am J Surg Pathol 1996;20:1161-1181.

13. Rugge M, Correa P, Dixon MF, et al. Gastric mucosal atrophy: interobserver consistency using new criteria for classification and grading. Aliment Pharmacol Ther 2002;16:1249-1259.

14. Kang JM, Kim N, Lee DH, et al. The effects of genetic polymorphisms of IL-6, IL-8, and IL-10 on Helicobacter pylori-induced gastroduodenal diseases in Korea. J Clin Gastroenterol 2009;43:420428 .

15. Rad R, Dossumbekova A, Neu B, et al. Cytokine gene polymorphisms influence mucosal cytokine expression, gastric inflammation, and host specific colonisation during Helicobacter pylori infection. Gut 2004;53:1082-1089. 
16. Zhang DL, Zheng HM, Yu BJ, Jiang ZW, Li JS. Association of polymorphisms of IL and CD14 genes with acute severe pancreatitis and septic shock. World J Gastroenterol 2005;11:4409-4413.

17. Ohyauchi M, Imatani A, Yonechi M, et al. The polymorphism interleukin 8-251 A/T influences the susceptibility of Helicobacter pylori related gastric diseases in the Japanese population. Gut 2005;54: 330-335.

18. Heinzmann A, Ahlert I, Kurz T, Berner R, Deichmann KA. Association study suggests opposite effects of polymorphisms within IL8 on bronchial asthma and respiratory syncytial virus bronchiolitis. J Allergy Clin Immunol 2004;114:671-676.

19. Hwang IR, Hsu PI, Peterson LE, et al. Interleukin-6 genetic polymorphisms are not related to Helicobacter pylori-associated gastroduodenal diseases. Helicobacter 2003;8:142-148.

20. Kato M, Saito M, Fukuda S, et al. ${ }^{13} \mathrm{C}$-Urea breath test, using a new compact nondispersive isotope-selective infrared spectrophotometer: comparison with mass spectrometry. J Gastroenterol 2004;39:629634.

21. Asaka M, Kato M, Takahashi S, et al. Guidelines for the management of Helicobacter pylori infection in Japan: 2009 revised edition. Helicobacter 2010;15:1-20.

22. Lacy BE, Talley NJ, Locke GR 3rd, et al. Review article: current treatment options and management of functional dyspepsia. Aliment Pharmacol Ther 2012;36:3-15.

23. Lee EJ, Gham CW, Park TW, et al. The effect of Helicobactor pylori eradication on the improvement of the symptoms in patients with functional dyspepsia and peptic ulcer disease. Korean J Med 2006; 71:141-148.

24. Kim SH, Hong DY, Kang PS, et al. Community-based Helicobacter pylori screening and its effects on eradication in patients with dyspepsia. Korean J Prev Med 2000;33:285-298.

25. Hong SJ, Sung IK, Kim JG, et al. Failure of a randomized, double-blind, placebo-controlled study to evaluate the efficacy of $H$. pylori eradication in $H$. pylori-infected patients with functional dyspepsia. Gut Liver 2011;5:468-471.

26. Suzuki H, Masaoka T, Sakai G, Ishii H, Hibi T. Improvement of gastrointestinal quality of life scores in cases of Helicobacter pylori-positive functional dyspepsia after successful eradication therapy. J Gastroenterol Hepatol 2005;20:1652-1660.

27. Talley NJ, Janssens J, Lauritsen K, Rácz I, Bolling-Sternevald E. Eradication of Helicobacter pylori in functional dyspepsia: randomised double blind placebo controlled trial with 12 months' follow up. The Optimal Regimen Cures Helicobacter Induced Dyspepsia (ORCHID) Study Group. BMJ 1999;318:833-837.

28. Suzuki H, Nishizawa T, Hibi T. Can Helicobacter pylori-associated dyspepsia be categorized as functional dyspepsia? J Gastroenterol Hepatol 2011;26(suppl 3):42-45.

29. Suzuki H, Matsuzaki J, Hibi T. What is the difference between Helicobacter pylori-associated dyspepsia and functional dyspepsia? J Neurogastroenterol Motil 2011;17:124-130.

30. Sugano K. Should we still subcategorize helicobacter pylori-associated dyspepsia as functional disease? J Neurogastroenterol Motil 2011; 17:366-371.
31. Iijima K, Ohara S, Sekine $\mathrm{H}$, et al. Changes in gastric acid secretion assayed by endoscopic gastrin test before and after Helicobacter pylori eradication. Gut 2000;46:20-26.

32. Mahadeva S, Goh KL. Epidemiology of functional dyspepsia: a global perspective. World J Gastroenterol 2006;12:2661-2666.

33. Winthorst WH, Post WJ, Meesters Y, Penninx BW, Nolen WA. Seasonality in depressive and anxiety symptoms among primary care patients and in patients with depressive and anxiety disorders; results from the Netherlands Study of Depression and Anxiety. BMC Psychiatry 2011;11:198.

34. Miwa H, Ghoshal UC, Gonlachanvit S, et al. Asian consensus report on functional dyspepsia. J Neurogastroenterol Motil 2012;18:150168.

35. Lane JA, Murray LJ, Harvey IM, Donovan JL, Nair P, Harvey RF. Randomised clinical trial: Helicobacter pylori eradication is associated with a significantly increased body mass index in a placebo-controlled study. Aliment Pharmacol Ther 2011;33:922-929.

36. Furuta T, Shirai N, Xiao F, Takashima M, Hanai H. Effect of Helicobacter pylori infection and its eradication on nutrition. Aliment Pharmacol Ther 2002;16:799-806.

37. Kim N, Lee SW, Kim JI, et al. Effect of Helicobacter pylori eradication on the development of reflux esophagitis and gastroesophageal reflux symptoms: a ationwide multi-center prospective Study. Gut Liver 2011;5:437-446.

38. Enck P, Vinson B, Malfertheiner P, Zipfel S, Klosterhalfen S. The placebo response in functional dyspepsia - reanalysis of trial data. Neurogastroenterol Motil 2009;21:370-377.

39. Oshima T, Toyoshima F, Nakajima S, Fukui H, Watari J, Miwa H. Genetic factors for functional dyspepsia. J Gastroenterol Hepatol 2011;26(suppl 3):83-87.

40. Yakoob J, Abid S, Abbas Z, et al. Distribution of Helicobacter pylori virulence markers in patients with gastroduodenal diseases in Pakistan. BMC Gastroenterol 2009;9:87.

41. Schilling D, Ott MG, Nilius M, et al. Specific Helicobacter pylori antigens unable to distinguish nonucler dyspepsia or peptic ulcer cases from asymptomatic seropositive controls: a nested case-control study in employees of a large company. Dig Dis Sci 2000;45:2444-2450.

42. Tan HJ, Rizal AM, Rosmadi MY, Goh KL. Role of Helicobacter pylori virulence factor and genotypes in non-ulcer dyspepsia. J Gastroenterol Hepatol 2006;21(1 Pt 1):110-115.

43. Toyoda K, Furusyo N, Ihara T, Ikezaki H, Urita Y, Hayashi J. Serum pepsinogen and Helicobacter pylori infection-a Japanese population study. Eur J Clin Microbiol Infect Dis 2012;31:2117-2124.

44. Locke CR 3rd, Talley NJ, Nelson DK, et al. Helicobacter pylori and dyspepsia: a population-based study of the organism and host. Am J Gastroenterol 2000;95:1906-1913.

45. Ebara S, Shirasaka D, Aoyama N, et al. The relationship between gastric emptying determined by the breath test and $H$. pylori. Hepatogastroenterology 2007;54:613-616.

46. Turkay C, Erbayrak M, Bavbek N, Yenidunya S, Eraslan E, Kasapoglu B. Helicobacter pylori and histopathological findings in patients with dyspepsia. Turk J Gastroenterol 2011;22:122-127. 